\title{
FOCUS ON EFFECTOR-TRIGGERED SUSCEPTIBILITY
}

\section{MPMI Focus Issue Overview}

Effector proteins play key roles in the molecular interplay between plants and plant-associated organisms, and effector biology remains one of the most active areas in the research field of molecular plant-microbe interactions. Using effectors as probes, much has been learned about pathogen virulence and host immunity, which has broad implications in developing diseaseresistant crops that are essential for global food security. Thus, the MPMI Editorial Board felt that it is an opportune time to showcase recent progress in this area.

The content in this focus issue reflects the diversity that is apparent in effector biology at multiple levels. At the organismal level, it is striking that every successful plant pathogen and pest produces effector proteins and relies on their function to cause disease. Much experimental attention has focused on effectors from bacteria, fungi, and oomycetes, which are at the forefront of this research area. However, effectors are also important for insects. Basu et al. review the herbivore-associated molecular patterns and effectors from the chewing insects and discuss their role in modulating plant defense. Analysis of salivary proteins by Jonckheere and coworkers reveal a novel effector family that is secreted by the herbivorous pest spider mite in a host-specific manner. In particular, the effector genes exhibit transcriptional plasticity when feeding on different hosts, suggesting specific host adaptation.

Viruses occupy the opposite end of the spectrum of organismal complexity but are known to produce quintessential effectors, in many cases with multiple functions. For example, the role of potyviral HcPro in suppression of host gene silencing is welldocumented, but Poque and colleagues describe an interaction between HcPro and an Arabidopsis salicylic acid (SA)-binding protein that indicates a direct interference of SA-mediated immunity by this viral effector. The tobamovirus replicase is another well-studied protein complex. Malpica-López et al. dissected the roles of two protein components in this complex and demonstrate their distinct functions in viral replication and suppression of host gene silencing.

Effectors are diverse in their sites of action. The plant extracellular space constitutes a major battleground in plant-pathogen interactions. Microbial pathogens produce an arsenal of effectors that operate in this matrix. The current knowledge of microbial apoplastic effectors is reviewed by Wang and Wang with an emphasis on the strategies to subvert plant immunity. Necrosis and ethylene-inducing peptide 1-like proteins (NLPs) are notable apoplastic effectors because they are represented in bacteria, fungi, and oomycetes, suggestive of important roles in virulence. However, Ahmad Azmi et al. demonstrate that the C-terminus of a Colletotrichum NLP can trigger immunity in cucurbits and restrict pathogen infection, pointing to the potential of apoplastic effectors as targets to develop resistance. Similarly, Hael-Conrad et al. provide evidence for an intriguing, systemic cell death that is elicited in Fragaria ananassa by a subtilisin-like protein produced by the fungal pathogen Acremonium strictum. Some of the first apoplastic effectors were described almost 30 years ago in the tomato leaf mold pathosystem. Mesarich and colleagues employ the cutting-edge approach of effectoromics to probe wild tomato accessions for new sources of host resistance triggered by fungal apoplastic effectors that may confer broad-spectrum resistance.

Delivered into host cells, cytoplasmic effectors directly manipulate plant cellular processes for the benefit of infection. However, plants have evolved intracellular NLR receptors to activate effectortriggered immunity. Białas et al. use three rice blast fungal effectors as examples to synthesize a dozen generally applicable lessons on cytoplasmic effectors, which are engaged in a constant arms race with NLR receptors during host-pathogen coevolution.

Diverse mechanisms have evolved for export of effectors, ranging from the insect stylet to the bacterial type III secretion system (T3SS). Scheibner et al. probe the factors that guide effector translocation through the T3SS by the bacterial pathogen Xanthomonas campestris. The Lu et al. describe a new, Arabidopsisbased pathosystem for the bacterial wilt pathogen Ralstonia solanacearum that can be used to dissect the functions of type III effectors (T3Es) and other virulence factors. One of the longestand best-studied T3Es is AvrPto produced by Pseudomonas syringae. Wu et al. describe a new virulence target of AvrPto with a direct connection to immunity activation by membrane-bound receptors upon perception of extracellular elicitors.

Diversity is also evident within the complex secretomes deployed by eukaryotic pathogens. This presents an experimental challenge, which is addressed in a review by Dalio et al. that provides a primer for prediction and functional characterization of effectors from fungi and oomycetes. Qi et al. employ several of these approaches, especially some of the heterologous expression systems, to characterize effector candidates from a destructive soybean rust pathogen for which few sources of resistance have been identified.

In closing, we are gratified by the strong response to the call for papers in this issue and sincerely thank the authors for contribution. Although we regret that not all accepted papers could be accommodated due to the space limit, upcoming issues of MPMI will feature additional effector-related papers on a variety of interesting topics, such as a Phytophthora effector that inhibits papain in papaya, a genomic/transcriptomic database for TAL effectors, interactions between Phytophthora effectors and citrus hosts, a functional comparison of conserved effectors from downy mildew and Phytophthora species, and the type III-dependent effector delivery from Salmonella spp. into plant cells. We look forward to these and other exciting research on effector biology in 2018 and are already planning the next Focus Issue on immunity triggered by effectors and microbe-associated molecular patterns to begin 2019.

Guest Editors Wenbo Ma and Yuanchao Wang MPMI Editor-in-Chief John McDowell 